\title{
Uso de internet móvil en Chile: explorando los antecedentes de su aceptación a nivel individual
}

\author{
Mobile internet usage in Chile: exploring the antecedents \\ of its acceptance at the individual level \\ Patricio Ramírez-Correa ${ }^{1}$ \\ Recibido 3 de septiembre de 2013, aceptado 22 de mayo de 2014 \\ Received: September 3, 2013 Accepted: May 22, 2014
}

\begin{abstract}
RESUMEN
El presente trabajo tiene por propósito la validación parcial de UTAUT 2 (Unified Theory of Acceptance and Use of Technology 2) en internet móvil. El estudio analiza la relación entre las variables motivación hedonista, relación precio/valor y hábito con la variable intención de uso de internet móvil en una muestra de usuarios chilenos. A partir de los datos recolectados en el estudio de campo se realizó un análisis SEM-PLS del modelo de investigación. Los resultados de este análisis muestran una sólida asociación tanto de la motivación hedonista y el hábito con la intención de uso de internet móvil. El modelo de investigación y los resultados de este análisis se exponen en este artículo.
\end{abstract}

Palabras clave: UTAUT 2, motivación hedonista, hábito, internet móvil, Chile.

\section{ABSTRACT}

The purpose of this paper is to accomplish a partial validation of UTAUT 2 (Unified Theory of Acceptance and Use of Technology 2) in mobile Internet. The study analyzes the relationship between hedonistic motivation, price/value and habit and the use intention of mobile internet in a sample of Chileans users. From the data collected in the field study, a SEM-PLS analysis of the research model was performed. The results of this analysis show a strong association of both hedonistic motivation and habit with the intention to use mobile internet. The research model and the results of this analysis are presented in this article.

Keywords: UTAUT 2, hedonistic motivation, habit, mobile internet, Chile.

\section{INTRODUCCIÓN}

En el contexto de las tecnologías de información (TI) Chile es el líder indiscutible en Latinoamérica, según el Indicador de la Sociedad de la Información [1]. Según este mismo índice, el 2011 Chile se posiciona como el primer país latinoamericano en ser clasificado mejor que un país de Europa. En este ámbito son destacables tanto el proceso de penetración de los teléfonos móviles, llegando a tener 1.227 unidades por cada 1.000 habitantes, como el actual número de usuarios de Internet, 480 por cada 1.000 habitantes [1].

En particular, e impulsado por la navegación en los teléfonos móviles smartphones, el uso de internet móvil ha tenido un crecimiento notable en Chile. El uso de internet móvil se refiere al acceso a internet desde un dispositivo móvil, como un smartphone, un teléfono o una tablet, conectado a una red de comunicaciones móvil. En diciembre de 2011 las conexiones tanto de banda ancha como de internet

1 Escuela de Ciencias Empresariales. Universidad Católica del Norte. Larrondo 1281. Coquimbo, Chile.

E-mail: patricio.ramirez@ucn.cl 
móvil 3G llegaron a 2.961.050, cifra que representa una variación del 104,8\% respecto de 2010 y de $126,3 \%$ respecto de 2009. Adicionalmente se proyecta que esta demanda por servicios móviles de conectividad crecerá 18 veces en los próximos cuatro años [2].

Por otra parte, la aceptación y uso de las TI a nivel individual es un fenómeno ampliamente estudiado en la literatura [3]. Internet móvil, como tecnología de consumo, no es la excepción a este interés científico, de hecho, recientes estudios se han orientado a modelar la aceptación y uso de esta tecnología en particular [4-8].

Debido a lo anterior, resulta relevante analizar los antecedentes del uso internet móvil en Chile a nivel individual. Considerando lo anterior, el propósito de este trabajo es la validación parcial de UTAUT 2 (Unified Theory of Acceptance and Use of Technology 2) en Chile. En particular, el estudio analizará la relación entre las variables motivación hedonista, relación precio/valor y hábito con la intención de uso de Internet móvil.

Tanto el crecimiento exponencial de las necesidades de TI en diversas áreas de la vida humana como el incremento de la tasa de fallas en la adopción de estas, instala a la predicción del uso de TI como un interesante área de estudio. Es por lo anterior que el desarrollo y validación en diversos contextos de modelos de adopción de TI a nivel individual es crucial en la sociedad actual. Es así que la originalidad y valor de la propuesta de UTAUT2 radica en el extender el modelo UTAUT al contexto de las TI de consumo.

El artículo se ha estructurado en la siguiente forma. Primero se presenta formalmente el modelo UTAUT 2 y sus orígenes. A continuación se expone el modelo parcial que será validado, detallando cada una de sus variables. Seguidamente se explica el trabajo de campo, finalizando con los resultados y conclusiones del estudio.

\section{REVISIÓN DE LA LITERATURA}

\section{Modelos de comportamiento humano}

La aceptación de tecnologías se enmarca dentro del estudio del comportamiento humano, tema que ha sido de interés científico durante mucho tiempo. En ese contexto, la teoría de la acción razonada (Theory of Reasoned Action, TRA) y la teoría del comportamiento planeado (Theory of Planned Behavior, TPB) [9-10] constituyen un marco conceptual útil para entender dicho comportamiento.

De acuerdo con TRA y TPB, el comportamiento de un individuo está determinado por la intención de realizar tal comportamiento, esta intensión es una función de la actitud y de las normas subjetivas, que se remontan a las creencias sobre el comportamiento y a las creencias normativas, respectivamente. $\mathrm{La}$ actitud representa los sentimientos positivos o negativos de un individuo sobre la realización de un comportamiento determinado, mientras que la intención describe la fuerza del propósito de realizar un comportamiento determinado [11]. Por otra parte, las creencias sobre el comportamiento se refieren a la evaluación positiva o negativa de la realización de un cierto comportamiento por parte del individuo, $\mathrm{y}$ las creencias normativas representan la percepción individual a las presiones sociales para realizar o no realizar un comportamiento. Los pesos relativos de estos dos tipos de creencias pueden variar entre los individuos [9].

\section{Modelos de aceptación de tecnología}

Davis [12] desarrolló el modelo de aceptación de tecnología (Technology Acceptance Model, TAM). TAM está influenciado por el marco conceptual establecido por TRA y TPB, y su propósito es explicar el proceso de aceptación de la TI a nivel individual. El enfoque principal de David estaba en dos elemento teóricos, la utilidad percibida y la facilidad de uso percibida, a los cuales señala como determinantes fundamentales para explicar la intención de comportamiento del usuario de utilizar el potencial de una innovación tecnológica, y en particular, de aceptar o rechazar una TI. La facilidad de uso percibida describe el grado de facilidad asociada al uso de la tecnología, y la utilidad percibida indica el grado en que una persona cree que el uso de la tecnología le ayudará a obtener mejoras en su desempeño. Concretamente, TAM indica que la intención individual de uso de una tecnología de la información es una función de la facilidad de uso observada y de la utilidad percibida. Además, TAM señala que la facilidad de uso advertida afecta directamente a la utilidad notada. 
Desde su propuesta, TAM ha sido ampliamente validado por la literatura científica, y en particular en forma exitosa en contextos internet [13-14].

Adicionalmente, diversos estudios han ampliado el modelo original de TAM. Venkatesh y Davis [15] desarrollaron una extensión teórica de TAM que explica la utilidad percibida y la intención de uso en términos de la influencia social (normas subjetivas, voluntariedad e imagen) y los procesos cognitivos instrumentales (relevancia para trabajo, calidad de las salidas, demostrabilidad del resultado y facilidad de uso percibida). Este modelo extendido de TAM es conocido como TAM 2. Posteriormente y a partir de un extenso análisis del uso de TAM, Venkatesh y Bala [3] proponen TAM 3, una extensión de TAM 2 que describe un conjunto de determinantes de la percepción de utilidad.

\section{Modelo UTAUT 2}

En forma adicional, en la literatura se encuentra UTAUT (Unified Theory of Acceptance and Use of Technology), un modelo que intenta explicar en forma global por qué los individuos utilizan las TI que tienen a su disposición. UTAUT fue desarrollado por Venkatesh, Morris y Davis [16] como una síntesis de la investigación sobre la aceptación de tecnologías disponibles. UTAUT propone cuatro constructos (la expectativa de rendimiento, la expectativa de esfuerzo, la influencia social y las condiciones de las facilidades) que influyen en la intención de uso y/o el uso de la tecnología.

Es necesario indicar que además de los modelos TRA, TPB, y TAM detallados anteriormente, el desarrollo de UTAUT se basa en otras alternativas propuestas para explicar el uso de tecnologías, como el Modelo Motivacional (Motivational Model, MM), el Modelo de Utilización de Computadores Personales (Model of PC Utilization, MPCU), la Teoría de Difusión de las Innovaciones (Innovation Diffusion Theory, IDT), y la Teoría Cognitiva Social (Social Cognitive Theory, SCT) [16]. Si bien el uso de UTAUT es ampliamente aceptado, también los modelos alternativos señalados han sido explorados por algunos autores.

Con el propósito de adaptar UTAUT al contexto del uso de tecnología de consumo, Venkatesh Venkatesh, Thong y Xin [4] proponen UTAUT 2. UTAUT 2 integra como antecedentes de la intención de uso de la tecnología los constructos motivación hedonista, relación precio / valor y hábito.

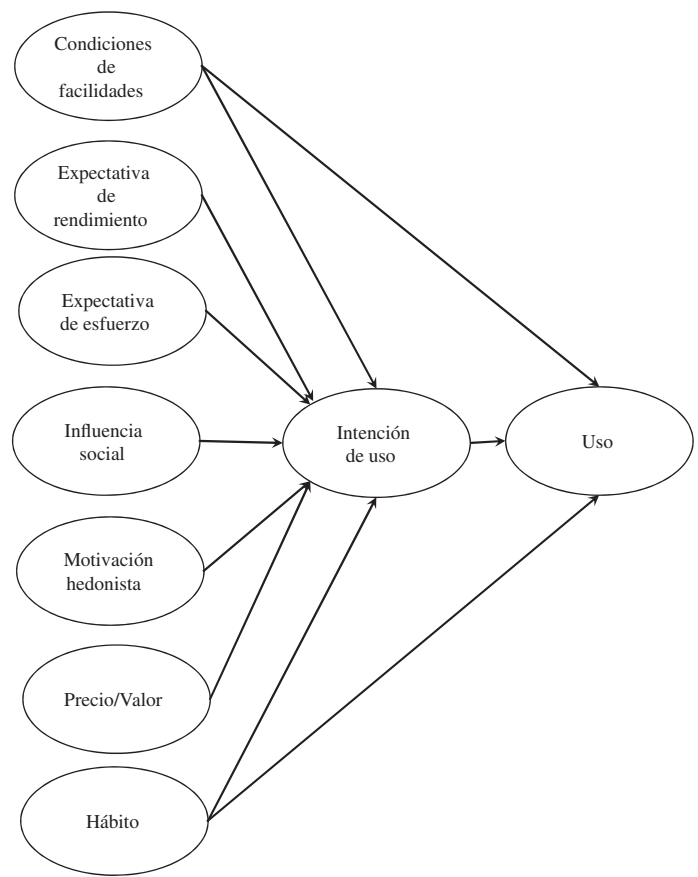

Figura 1. Modelo UTAUT 2.

La Figura 1 muestra el modelo UTAUT 2. Este modelo se puede explicar como sigue. Al aumentar la expectativa de rendimiento, la expectativa de esfuerzo, la influencia social, las condiciones de las facilidades, la motivación hedonista, la relación precio / valor o del hábito, aumentará la intención de uso. Adicionalmente, un aumento de la intención de uso, las condiciones de las facilidades o del hábito, aumentará directamente el uso de la tecnología de consumo.

Debido a la novedad de UTAUT2, las referencias a este modelo en la literatura actual están asociadas principalmente con las variables que comparte con el modelo UTAUT (expectativa de rendimiento, expectativa de esfuerzo, influencia social, y condiciones de las facilidades), y solo unos pocos estudios utilizan las nuevas variables propuestas (motivación hedonista, relación precio/valor, y hábito).

\section{MODELO PROPUESTO E HIPÓTESIS}

\section{Modelo}

Atendiendo las características exploratorias de este trabajo se decidió explorar solo las nuevas relaciones propuestas en UTAUT 2. En particular, 
las relaciones entre la motivación hedonista, la relación precio / valor y el hábito con la intención de uso de la tecnología de consumo. No se incluyó la relación entre intención de uso y uso atendiendo las críticas en relación con modelos de aceptación de tecnologías que no miden directamente el uso.

La Figura 2 muestra el modelo de investigación utilizado en este estudio. En el modelo un aumento en la motivación hedonista, la valorización de la relación precio/valor o en el hábito incide positivamente en la intención de uso de la tecnología.

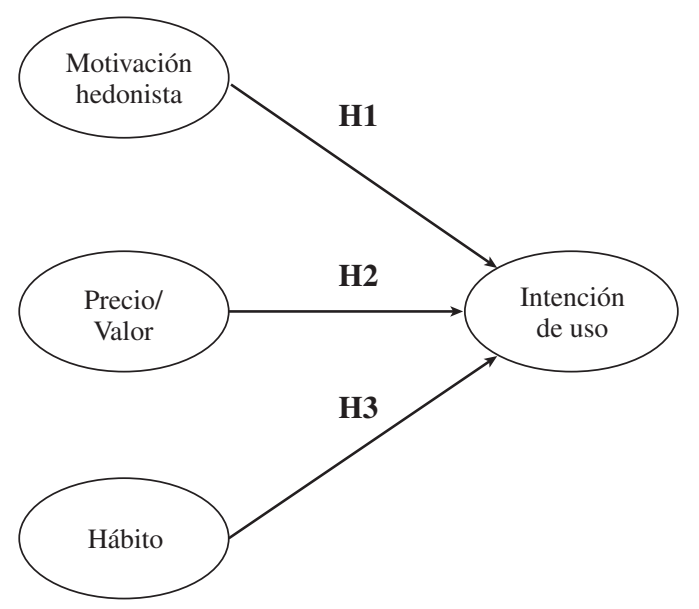

Figura 2. Modelo de investigación.

Basados en [4, 9], a continuación se definen las variables del modelo:

- Motivación hedonista (HM): La diversión o el placer que se deriva del uso de una tecnología.

- Precio/Valor (PV): Compensación cognitiva del consumidor entre los beneficios percibidos de las aplicaciones y el costo monetario del uso de una tecnología.

- Hábito (HT): Grado en que las personas tienden a realizar conductas de forma automática debido al aprendizaje.

- Intención de uso (BI): Grado en que una persona ha formulado planes conscientes de usar o no usar en el futuro una tecnología.

\section{Hipótesis}

Basados en [4] se proponen las siguientes hipótesis:

- H1: Un aumento de la motivación hedonista aumenta la intención de uso de la tecnología de consumo.
- H2: Un aumento de la valorización de la relación precio/valor aumenta la intención de uso de la tecnología de consumo.

- H3: Un aumento del hábito aumenta la intención de uso de la tecnología de consumo.

\section{METODOLOGÍA}

\section{Muestra}

El trabajo de campo se realizó en mayo de 2012 mediante una encuesta a los usuarios del internet móvil en Chile. Los usuarios encuestados fueron mayoritariamente académicos, alumnos universitarios de pregrado a tiempo completo, y alumnos de postgrado a tiempo parcial de las ciudades de Santiago, Coquimbo y Antofagasta.

Luego de su procesamiento y validación se obtuvieron 133 encuestas válidas. Los individuos de la muestra tenían una edad promedio de 31 años y correspondían en $73,7 \%$ a hombres.

\section{Escalas}

Las escalas de medición aplicadas para medir los constructos o variables latentes del modelo han sido probadas en la literatura $[4,9]$. Todas las escalas utilizadas fueron tipo Likert (siete puntos).

A continuación se detallan los ítems de cada escala.

1) Motivación hedonista (HM)

- HM1. Usar Internet móvil es divertido.

- HM2. El uso de Internet móvil es agradable.

- HM3. El uso de Internet móvil es muy entretenido.

2) Relación Precio/Valor (PV)

- PV1. Internet móvil tiene un precio razonable.

- PV2. Internet móvil entrega un buen valor por el dinero que cuesta.

- PV3. Al precio actual, Internet móvil ofrece un buen valor.

3) Hábito (HT)

- HT1. El uso de Internet móvil se ha convertido en un hábito para mí.

- HT2. Yo soy adicto a usar Internet móvil.

- HT3. Yo debo usar Internet móvil.

- HT4. El uso de Internet móvil se ha convertido en algo natural para mí.

4) Intención de uso (BI)

- BI1. Tengo la intención de seguir utilizando Internet móvil en el futuro. 
- BI2. Siempre voy a tratar de usar Internet móvil en mi vida diaria.

- BI3. Tengo planes de continuar el uso frecuente de Internet móvil.

\section{RESULTADOS}

Considerando el propósito exploratorio de este estudio y sus restricciones seleccionamos la técnica PLS (Partial Least Square) para contrastar las hipótesis de la investigación [17-19]. El software SmartPLS 2.0 de M3 fue utilizado para este análisis [20].

Tabla 1. Fiabilidad de los constructos.

\begin{tabular}{|c|c|c|c|}
\hline 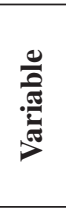 & $\sum_{2}^{1}$ & 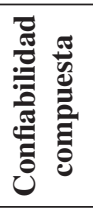 & 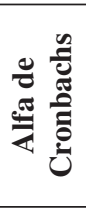 \\
\hline BI & 0,85 & 0,94 & 0,91 \\
\hline HT & 0,76 & 0,93 & 0,90 \\
\hline $\mathrm{HM}$ & 0,96 & 0,99 & 0,98 \\
\hline PV & 0,87 & 0,95 & 0,93 \\
\hline
\end{tabular}

En una primera fase se valoró el modelo de medida, en ella confirmamos tanto fiabilidad individual de cada ítem y constructo (ver Tablas 1 y 2) como validez convergente y validez discriminante de todos los constructos (ver Tabla 3).

En una segunda fase procedimos a evaluar si el modelo estructural apoya al modelo de investigación propuesto.

Tabla 2. Cargas cruzadas de los ítems.

\begin{tabular}{|c|c|c|c|c|}
\hline & $\ddot{\theta}$ & $\sum$ & 夏 & 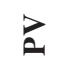 \\
\hline BI1 & $\mathbf{0 , 8 9}$ & 0,68 & 0,42 & 0,46 \\
\hline BI2 & 0,94 & 0,58 & 0,60 & 0,44 \\
\hline BI3 & $\mathbf{0 , 9 3}$ & 0,68 & 0,43 & 0,26 \\
\hline HM1 & 0,69 & 0,98 & 0,12 & 0,20 \\
\hline HM2 & 0,66 & 0,98 & 0,08 & 0,21 \\
\hline HM3 & 0,70 & 0,98 & 0,20 & 0,25 \\
\hline HT1 & 0,27 & $-0,02$ & 0,84 & 0,48 \\
\hline HT2 & 0,40 & 0,07 & 0,88 & 0,44 \\
\hline HT3 & 0,61 & 0,23 & 0,89 & 0,41 \\
\hline HT4 & 0,42 & 0,09 & 0,88 & 0,44 \\
\hline PV1 & 0,36 & 0,10 & 0,51 & 0,97 \\
\hline PV2 & 0,50 & 0,37 & 0,43 & 0,96 \\
\hline PV3 & 0,20 & 0,02 & 0,49 & 0,87 \\
\hline
\end{tabular}

Tabla 3. Correlaciones de constructos ${ }^{\mathrm{a}}$.

\begin{tabular}{|l|c|c|c|c|}
\hline & $\overrightarrow{\boldsymbol{x}}$ & $\mathbf{\Xi}$ & $\grave{i}$ & $\vec{\Xi}$ \\
\hline BI & $\mathbf{0 , 9 2}$ & - & - & - \\
\hline HT & 0,52 & $\mathbf{0 , 8 7}$ & - & - \\
\hline HM & 0,70 & 0,14 & $\mathbf{0 , 9 8}$ & - \\
\hline PV & 0,42 & 0,50 & 0,23 & $\mathbf{0 , 9 4}$ \\
\hline
\end{tabular}

(a) En la diagonal raíz de AVE del constructo.

La Figura 3 muestra los caminos estructurales con valores significativos y el valor de los R2. La Tabla 4 muestra el resultado del proceso de bootstrap para el cálculo de la fiabilidad de los caminos estructurales.

Tal como muestra la Tabla 4, los resultados confirman dos de las relaciones que se establecieron en el modelo de investigación. El valor beta de la relación entre PV y BI no permite apoyar esta causalidad (beta menor a 0,2 ).

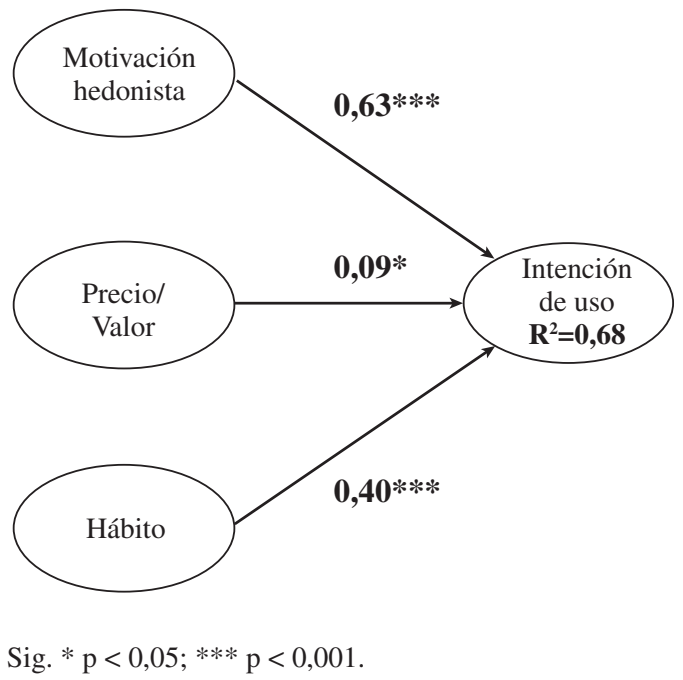

Figura 3. Modelo valorado.

Tabla 4. Coeficientes de los caminos (paths).

\begin{tabular}{|c|c|c|c|}
\hline Еี & ڤ్ & 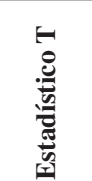 & $\stackrel{\circ 0}{\circ}$ \\
\hline HT $->$ BI & 0,40 & 9,10 & $* * *$ \\
\hline $\mathrm{HM}->\mathrm{BI}$ & 0,63 & 11,41 & $* * *$ \\
\hline $\mathrm{PV}->\mathrm{BI}$ & 0,08 & 2,35 & $*$ \\
\hline
\end{tabular}


Finalmente se calculó como medida de ajuste global (Goodness of Fitness, GoF) la raíz cuadrada de la multiplicación del promedio de los $\mathrm{R}$ cuadrados y el promedio de los AVE, su resultado de 0,77 indica que el modelo estructural puede predecir de buena forma la intención de uso de tecnologías de consumo.

A continuación, en la Tabla 5 se resume el contraste de hipótesis.

Tabla 5. Resumen contraste de hipótesis.

\begin{tabular}{|c|c|}
\hline Hipótesis & Contraste \\
\hline $\begin{array}{l}\text { H1: Un aumento de la motivación he- } \\
\text { donista aumenta la intención de uso } \\
\text { de la tecnología de consumo. }\end{array}$ & Soportada \\
\hline $\begin{array}{l}\text { H2: Un aumento de la valorización } \\
\text { de la relación precio/valor aumenta la } \\
\text { intención de uso de la tecnología de } \\
\text { consumo. }\end{array}$ & $\begin{array}{c}\text { No } \\
\text { soportada }\end{array}$ \\
\hline $\begin{array}{l}\text { H3: Un aumento del hábito aumenta } \\
\text { la intención de uso de la tecnología de } \\
\text { consumo. }\end{array}$ & Soportada \\
\hline
\end{tabular}

\section{CONCLUSIONES Y LIMITACIONES}

Este estudio logró validar en forma parcial el modelo UTAUT 2 en el uso de una tecnología de consumo. El modelo había sido previamente validado en Hong Kong y la originalidad del presente trabajo está en su aplicación en Chile, siguiendo las sugerencias de investigaciones futuras de los autores originales [4]. En particular, el estudio analizó las relaciones entre las variables motivación hedonista, la relación precio/valor y el hábito, con la variable intención de uso de internet móvil.

Desde la perspectiva metodológica se desea destacar la alta fiabilidad de las escalas de medida y la excelente medida de ajuste global del modelo estructural propuesto.

Desde la perspectiva práctica se destaca el gran impacto de la motivación hedonista en la intención de uso de la Internet móvil. Asimismo, es importante subrayar la relación positiva entre el hábito, es decir, el grado de realizar conductas de forma automática debido al aprendizaje, y la intención de uso de internet móvil. Luego, los usuarios de Internet móvil tendrán mayor intención del uso de esta tecnología si aprenden y disfrutan su uso.
Por otra parte, es destacable la baja relación existente entre la relación precio/calidad y la intención de uso de Internet móvil. Luego, un aumento o disminución en la percepción del consumidor de esta variable de consumo no implica un efecto mayor en su intención de usar Internet móvil. Una explicación posible se puede asociar a los altos costos de acceso de Internet móvil en Chile, lo que sesga la muestra por conveniencia del estudio a individuos con poder adquisitivo mayor al promedio, y que por lo mismo son menos sensibles a esta variable.

Este estudio tiene limitaciones que se espera superar en trabajos futuros. Primero, el tamaño de la muestra y el método de selección hacen imposible extrapolar los resultados a un universo mayor. Segundo, debido a las restricciones presupuestarias no se validó el modelo completo, perdiendo el análisis de las relaciones estudiadas en un contexto mayor. Y finalmente, no se pudo segmentar a los usuarios ni por proveedor ni por tipo de dispositivo para acceder de internet móvil, creemos que sería de utilidad realizar esta segmentación en una próxima investigación.

\section{REFERENCIAS}

[1] Everis/IESE. "Indicador de la Sociedad de la Información (ISI) - Everis/IESE, Situación de las Tecnologías de la Información en Latinoamérica, la Unión Europea y EE.UU.”. Especial referencia a los casos de Argentina, Brasil, Chile, Colombia, México y Perú". 2012.

[2] SUBTE. "Encuestas sobre Acceso, Uso y Usuarios y Disposición de Pago por Internet 2011”. Subsecretaría de Telecomunicaciones. Ministerio de Transportes y Telecomunicaciones. Gobierno de Chile. 2012.

[3] V. Venkatesh and H. Bala. "Technology Acceptance Model 3 and a Research Agenda on Interventions". Decision Sciences. Vol. 39, Issue 2, pp. 273-315. 2008.

[4] V. Venkatesh, J. Thong and X. Xin. "Consumer Acceptance and Use of Information Technology: Extending the Unified Theory of Acceptance and Use of Technology". MIS Quarterly. Vol. 36, Issue 2, pp. 157-178. 2012. [5] S. Mardikyan, B. Besiroglu and G. Uzmaya. "Behavioral Intention towards the Use of 
3G Technology". Communications of the IBIMA, pp. 1-10, 2012.

[6] S.Y. Park, M.-W. Nam and S.-B. Cha. "University students' behavioral intention to use mobile learning: Evaluating the technology acceptance model". British Journal of Educational Technology. Vol. 43, Issue 4, pp. 592-605. 2012.

[7] Y. Liu and H. Li. "Mobile internet diffusion in China: an empirical study". Industrial Management \& Data Systems. Vol. 110, Issue 3, pp. 309-324. 2010.

[8] S. Kurnia, S.P. Smith and H. Lee. "Consumers' perception of mobile internet in Australia". E-Business Review. Vol. 5, Issue 1, pp. 19-32. 2006.

[9] I. Ajzen and M. Fishbein. "Understanding Attitudes and Predicting Social Behavior". Prentice Hall, Englewood Cliffs, NJ, USA. 1980.

[10] I. Ajzen. "From intentions to actions: a theory of planned behavior". In: J. Kuhl and J. Beckman (Eds.). Action-Control: From Cognition to Behavior. Springer-Verlag. 1985.

[11] M. Fishbein and I. Ajzen. "Belief Attitude, Intention and Behavior: An Introduction to Theory and Research". Addison-Wesley, Reading, MA, USA. 1975.

[12] F.D. Davis. "Perceived Usefulness, Perceived Ease of Use, and Usage of Information Technology: A Replication". MIS Quarterly. Vol. 13, Issue 3, pp. 319-339. 1989.

[13] P. Ramírez-Correa, J. Arenas-Gaitán y F.J. Rondán-Cataluña. "Uso de los servicios de redes sociales por la generación Y". Ingeniare. Revista chilena de ingeniería. Vol. $20 \mathrm{~N}^{\circ} 3$, pp. 425-432. 2012.

[14] J. Arenas-Gaitán, P. Ramírez-Correa and F.J. Rondán-Cataluña. "Cross cultural analysis of the use and perceptions of web based learning systems". Computers and Education. Vol. 57, Issue 2, pp. 1762-1774. 2011.

[15] V. Venkatesh and F.D. Davis. "A Theoretical Extension of the Technology Acceptance Model: Four Longitudinal Field Studies". Management Science. Vol. 46, Issue 2, pp. 186-204. 2000.

[16] V. Venkatesh, M.G. Morris and G.B. Davis. "User Acceptance of Information Technology: Toward a Unified View". MIS Quarterly. Vol. 27, Issue 3, pp. 425-478. 2003.

[17] W.W. Chin. "The partial least squares approach for structural equation modeling". In G.A. Marcoulides (Ed.). Modern Methods for Business Research, Lawrence Erlbaum Associates. USA. 1998.

[18] M. Tenenhaus, V.E. Vinzi, Y-M. Chatelin and C. Lauro. "PLS path modeling". Computational Statistics and Data Analysis. Vol. 48, pp. 159-205. 2005.

[19] C.M. Ringle, S. Wende and A. Will. "SmartPLS 2.0 (M3) beta". Hamburg, Alemania. 2005. Date of visit: May 10, 2012. URL: http://www.smartpls.de

[20] C. Fornell and D.F. Larcker. "Evaluating structural equation models with unobservable variables and measurement error". Journal of Marketing Research. Vol. 18, Issue 1, pp. 39-50. 1981. 